\title{
RADIATIVE AND AUGER DECAY OF K-VACANCY LEVELS IN THE Ne, Mg, Si, S, Ar, AND Ca ISONUCLEAR SEQUENCES
}

\author{
P. PAlmeri And P. Quinet ${ }^{1}$ \\ Astrophysique et Spectroscopie, Université de Mons-Hainaut, B-7000 Mons, Belgium; patrick.palmeri@umh.ac.be, pascal.quinet@umh.ac.be
}

C. Mendoza and M. A. Bautista

Centro de Física, IVIC, Caracas 1020A, Venezuela; claudio@ivic.ve, mbautist@ivic.ve

AND

J. García And T. R. Kallman

NASA Goddard Space Flight Center, Greenbelt, MD 20771; javier@milkyway.gsfc.nasa.gov, timothy.r.kallman@nasa.gov Received 2007 September 25; accepted 2008 February 18

\begin{abstract}
The HFR and AUTOSTRUCTURE atomic structure codes are used to compute complete data sets of level energies, wavelengths, $A$-values, and radiative and Auger widths for $\mathrm{K}$-vacancy states of the $\mathrm{Ne}, \mathrm{Mg}, \mathrm{Si}, \mathrm{S}, \mathrm{Ar}$, and Ca isonuclear sequences. Ions with electron number $N>9$ are treated for the first time. Detailed comparisons with previous measurements and theoretical data for ions with $N \leq 9$ are carried out in order to estimate reliable accuracy ratings.
\end{abstract}

Subject headings: atomic processes — atomic data — line: formation — X-rays: general

Online material: machine-readable tables

\section{INTRODUCTION}

While X-ray emission is characteristic of hot $\left(T \geq 10^{6} \mathrm{~K}\right)$ plasmas, X-ray spectra can also be used to study plasmas of lower ionization via inner-shell transitions. Processes which preferentially remove inner-shell electrons, such as photoionization or collisions with suprathermal particles, can give rise to emission of inner-shell fluorescence lines or K-edge absorption in astrophysical spectra. These features are observed from several important classes of astrophysical sources, being potential diagnostics of their conditions: ionization, gas motions, abundances, and innershell ionization rate. The grating instruments on the Chandra $X$-Ray Observatory and XMM-Newton space observatory have provided high signal-to-noise ratio spectra of these features, with typical energy resolution of $\varepsilon / \Delta \varepsilon \simeq 500-1000$. Exploitation of these diagnostics has been limited by the accuracy of the atomic data for these transitions (energies, cross sections, and lifetimes). This paper is part of a large effort to calculate as many of these data as possible with reliable accuracy using available computational methods. Previous papers in this series have focussed on the data for iron and oxygen; in this and subsequent papers we concentrate on medium-Z elements, namely $\mathrm{Ne}, \mathrm{Mg}, \mathrm{Si}, \mathrm{S}, \mathrm{Ar}$, and $\mathrm{Ca}$.

In the X-ray spectra obtained with Chandra and XMM-Newton, $\mathrm{K}$ lines and edges from medium- $Z$ elements have been observed from compact objects in which photoionization is thought to be the dominant ionization mechanism, and from low-ionization material which is seen along the line of sight to various bright sources. The active galactic nucleus (AGN) NGC 3783 observed by Kaspi et al. (2002) shows K-absorption lines of $\mathrm{H}$ - and $\mathrm{He}$ like $\mathrm{Ne}, \mathrm{Mg}$, Si, S, possibly $\mathrm{Ar}$, and $\mathrm{Ca}$, and from lower stages of ionization of Si and S. They are found to be blueshifted and asymmetric, and no correlation is obtained between the velocity shifts and the ionization potential of the ions. Absorption from

\footnotetext{
${ }^{1}$ Also at: IPNAS, Sart Tilman B15, Université de Liège, B-4000 Liège, Belgium.
}

low and medium stages of $\mathrm{Si}$ and $\mathrm{S}$ are also seen in the spectra from AGNs MCG-6-30-15 (Lee et al. 2001) and IRAS $13349+2438$ (Holczer et al. 2007), and from the X-ray binary Cyg X-1 (Chang \& Cui 2007). In emission these lines are seen in the spectra from Vela X-1 by Watanabe et al. (2006) and 4U1700-37 by Boroson et al. (2003). Furthermore, it is likely that future observations, which provide high-resolution X-ray spectra from spatially extended sources, will reveal inner-shell features in supernova remnants and stellar coronae. In these sources, such features may indicate existence of nonthermal particles or nonequilibrium ionization, and their analysis requires accurate data for the wavelengths and other atomic quantities associated with the $\mathrm{K}$ lines from medium- $Z$ elements.

Following the work by Palmeri et al. (2002, 2003a, 2003b), Bautista et al. (2003, 2004), Mendoza et al. (2004), and Kallman et al. (2004) on the K lines of Fe and by García et al. (2005) on the K-shell photoabsorption of $\mathrm{O}$ ions, we report new atomic data for K-vacancy levels in the $\mathrm{Ne}, \mathrm{Mg}, \mathrm{Si}, \mathrm{S}, \mathrm{Ar}$, and $\mathrm{Ca}$ isonuclear sequences. Prime objectives are to improve the atomic database of the XSTAR modeling code (Bautista \& Kallman 2001) and to prepare ionic targets (configuration expansions and orbitals) for the lengthy computations of the photoabsorption cross sections where both radiative and Auger dampings are key effects. In this respect, available atomic structure data sets - namely K-vacancy level energies, wavelengths, $A$-values, and Auger widths-for first-row ions with electron number $3 \leq N \leq 10$ are far from complete while for the second row $(11 \leq N \leq 20)$ they hardly exist.

Previous work on the K-shell structure of medium- $Z$ elements includes that by Faenov et al. (1994) on the satellites of the He-like resonance line in species with atomic number $12 \leq Z \leq$ 16 and electron number $4 \leq N \leq 9$. They have measured wavelengths in a $\mathrm{CO}_{2}$ laser produced plasma and computed wavelengths, $A$-values, and Auger rates with the MZ code (Vainshtein \& Safronova 1978, 1980). Biémont et al. (2000) have measured wavelengths for Ar ions with $3 \leq N \leq 9$ in a plasma focus discharge, providing also theoretical wavelengths, $A$-values, and 
Auger rates calculated with both the HFR atomic structure code (Cowan 1981) and the multiconfiguration Dirac-Fock (MCDF) YODA code of Hagelstein \& Jung (1987). K-vacancy level energies, wavelengths, $A$-values, and Auger and radiative widths have been computed for ions of the beryllium $(6 \leq Z \leq 26)$, boron $(6 \leq Z \leq 54)$, and carbon $(6 \leq Z \leq 54)$ isoelectronic sequences with the MCDF method by Chen (1985), Chen \& Crasemann (1988), and Chen et al. (1997), respectively. Inner-shell excitation energies and autoionization rates for ions with $6 \leq Z \leq 54$ and $6 \leq N \leq 9$ have been computed by Safronova \& Shlyaptseva (1999) using the $1 / Z$ perturbation theory method. Behar \& Netzer (2002) have calculated with the relativistic multiconfiguration HULLAC code (Bar-Shalom et al. 2001) wavelengths and oscillator strengths for the $1 s-n p$ transitions $(n \leq 3)$ in ions of $\mathrm{Ne}$, $\mathrm{Mg}, \mathrm{Al}, \mathrm{Si}, \mathrm{S}, \mathrm{Ar}$, and Ca with $2 \leq N \leq 9$. Deslattes et al. (2003) have produced a comprehensive compilation of both measured and theoretical transition energies for $\mathrm{K}$ lines and edges in elements with $10 \leq Z \leq 100$. Gorczyca et al. (2003) have audited the fluorescence database by Kaastra \& Mewe (1993) which is widely used in modeling codes, in particular their scaling procedures along isoelectronic sequences. They have found serious flaws which appear to compromise the application of this database in plasma modeling.

The outline of the present report is as follows. The numerical methods are briefly described in $\S 2$, while an analysis of the results based on comparisons with previous experimental and theoretical values is carried out in $\S 3$. The two supplementary electronic tables are explained in $\S 4$, while some conclusions are finally discussed in $\S 5$.

\section{NUMERICAL METHODS}

The numerical approach has been fully described by Bautista et al. (2003). The atomic data are computed with the structure codes HFR (Cowan 1981) and AUTOSTRUCTURE (Eissner et al. 1974; Badnell 1986, 1997). Wave functions are calculated with the Breit-Pauli relativistic corrections,

$$
H_{\mathrm{bp}}=H_{\mathrm{nr}}+H_{1 \mathrm{~b}}+H_{2 \mathrm{~b}},
$$

where $H_{\mathrm{nr}}$ is the usual nonrelativistic Hamiltonian. The one-body relativistic operators,

$$
H_{1 \mathrm{~b}}=\sum_{n=1}^{N} f_{n}(\text { mass })+f_{n}(\mathrm{~d})+f_{n}(\mathrm{so})
$$

represent the spin-orbit interaction, $f_{n}$ (so), and the non-finestructure mass variation, $f_{n}$ (mass), and the one-body Darwin correction, $f_{n}(\mathrm{~d})$. The two-body Breit operators are given by

$$
H_{2 \mathrm{~b}}=\sum_{n<m} g_{n m}(\mathrm{so})+g_{n m}(\mathrm{ss})+g_{n m}(\mathrm{css})+g_{n m}(\mathrm{~d})+g_{n m}(\mathrm{oo})
$$

where the fine-structure terms are $g_{n m}$ (so) (spin-other-orbit and mutual spin-orbit) and $g_{n m}(\mathrm{ss})$ (spin-spin), and the non-finestructure counterparts are $g_{n m}(\mathrm{css})$ (spin-spin contact), $g_{n m}(\mathrm{~d})$ (two-body Darwin), and $g_{n m}(\mathrm{oo})$ (orbit-orbit). Core relaxation effects are studied with AUTOSTRUCTURE by comparing ion representations where all the electron configurations have a common basis of orthogonal orbitals, to be referred to hereafter as approximation AS1, with one where each configuration has its own basis, approximation AS2. HFR computes energies, $A$-values, and Auger rates with bases of nonorthogonal orbitals obtained by optimizing the energy of each configuration and neglects the

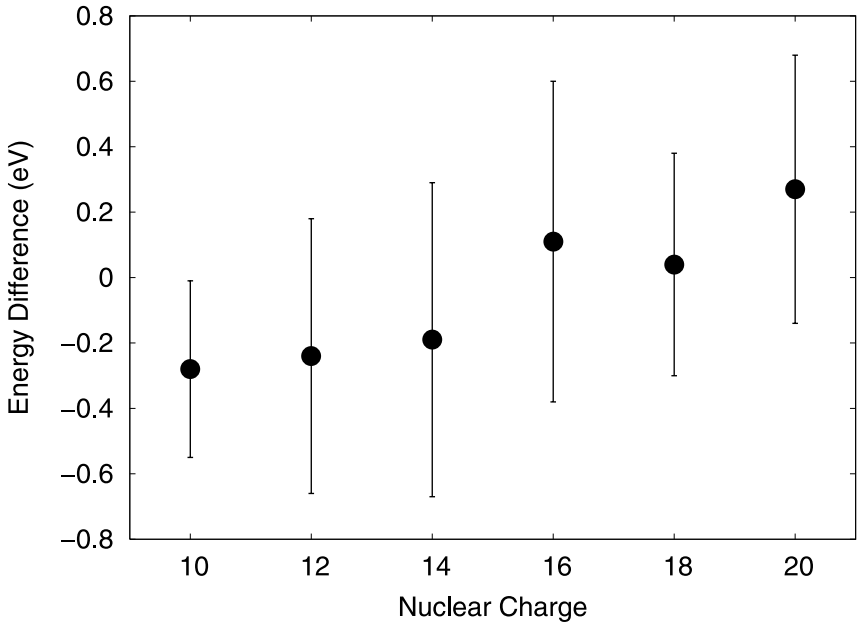

FIG. 1.- Average differences between the HFR1 energies and those in the NIST database ver. 3.1.2 (Ralchenko et al. 2007) for the K-vacancy levels in the $\mathrm{He}$ and $\mathrm{Li}$ isoelectronic sequences. Error bars indicate the standard deviation. For nuclear charge $Z=18$, the experimental level energies for the Li-like ion have been excluded as they are believed to be incorrect.

part of the Breit interaction (3) that cannot be reduced to a onebody operator. This data set is to be labeled HFR1.

\section{RESULTS}

We have carried out detailed comparisons with previous data in order to obtain accuracy estimates and detect weak points. In this respect, Palmeri et al. (2006) have already presented the outcome for the $\mathrm{S}$ isonuclear sequence. Their comparison with the experimental K-level energies for $\mathrm{S}$ xV and $\mathrm{S}$ xIV results in differences not larger than $1 \mathrm{eV}$ for HFR 1 and $2 \mathrm{eV}$ for AS2, but for the only level reported for S I $\left([1 s] 3 p^{5}{ }^{3} P_{2}^{o}\right)$, differences of $3.3 \mathrm{eV}$ and $3.6 \mathrm{eV}$ are respectively found. The level of agreement between HFR1 and AS2 reported by Palmeri et al. for the $\mathrm{S}$ sequence is $\sim 1 \mathrm{eV}$ for level energies, $\sim 2 \mathrm{~m} \AA$ for wavelengths, and $\sim 10 \%$ for radiative and Auger rates greater than $10^{13} \mathrm{~s}^{-1}$. They cannot explain, however, large discrepancies with the MCDF data by Chen et al. (1997) for C-like S XI. Since a similar degree of discord with Chen et al. was encountered by Palmeri et al. (2003a) for Fe xxI, we emphasize here comparisons with the experimental and theoretical data sets for Ar ions by Biémont et al. (2000) particularly those computed with the MCDF method, as they will provide more solid evidence on the questionable accuracy of the data by Chen et al.

\subsection{Energy levels}

With the exception of the He-, Li-, and Be-like species, there is a general lack of experimental $\mathrm{K}$-vacancy level energies for the isonuclear sequences under study here. This severely limits the possibilities of fine tuning the numerical methods in order to increase data accuracy and to provide reliable rankings. For this reason, we mainly base our approach on computations with the HFR code as it attains marginally more reliable ab initio energies due to the use of nonorthogonal basis sets for each electronic configuration.

In Figure 1 we show the average energy differences between the values listed in the NIST database version 3.1.2 (Ralchenko et al. 2007) and HFR1 for the K-vacancy levels in He- and Li-like ions. Differences are never larger than $1 \mathrm{eV}$ if those for Li-like Ar XVI are excluded, as the NIST K-vacancy level energies for this ion are believed to be grossly in error. As listed in Table 1, the energy difference for the $1 s 2 s^{2}{ }^{2} S_{1 / 2}$ level in $\mathrm{Ar}$ XVI is $0.1 \mathrm{eV}$ 
TABLE 1

K-Vacancy Level Energies in Ar xvi

\begin{tabular}{|c|c|c|}
\hline Level & $\begin{array}{l}\operatorname{Expt}^{\mathrm{a}} \\
(\mathrm{eV})\end{array}$ & $\begin{array}{c}\text { HFR1 } \\
(\mathrm{eV})\end{array}$ \\
\hline $1 s 2 s^{2}{ }^{2} S_{1 / 2} \ldots \ldots \ldots \ldots$ & 3079.0 & 3079.1 \\
\hline $1 s\left({ }^{2} S\right) 2 s 2 p\left({ }^{3} P^{o}\right){ }^{4} P_{1 / 2}^{o} \ldots \ldots \ldots$ & 3842.3 & 3086.7 \\
\hline 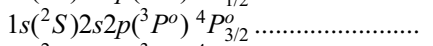 & 3843.8 & 3087.9 \\
\hline 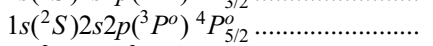 & 3847.1 & 3090.1 \\
\hline 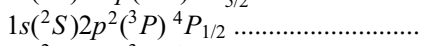 & 3876.2 & 3121.6 \\
\hline $1 s\left({ }^{2} S\right) 2 p^{2}\left({ }^{3} P\right){ }^{4} P_{3 / 2} \ldots \ldots \ldots \ldots \ldots \ldots \ldots$ & 3878.6 & 3123.0 \\
\hline $1 s\left({ }^{2} S\right) 2 p^{2}\left({ }^{3} P\right){ }^{4} P_{5 / 2} \ldots \ldots \ldots \ldots \ldots \ldots \ldots$ & 3881.0 & 3124.9 \\
\hline 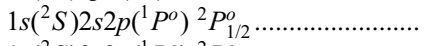 & 3884.8 & 3124.9 \\
\hline 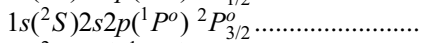 & 3885.9 & 3125.8 \\
\hline 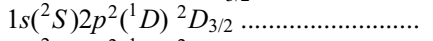 & 3896.6 & 3139.8 \\
\hline $1 s\left({ }^{2} S\right) 2 p^{2}\left({ }^{1} D\right)^{2} D_{5 / 2}$ & 3897.2 & 3140.4 \\
\hline
\end{tabular}

${ }^{\text {a }}$ NIST database V3.1.2 (Ralchenko et al. 2007) and T. Shirai et al. (1999, unpublished).

while those for the rest are $\sim 756 \mathrm{eV}$. The experimental inaccuracies for this ion are further established with the wavelength measurement by Beiersdorfer et al. (2002) for the $1 s 2 s 2 p^{4} P_{5 / 2}^{o} \rightarrow$ $1 s^{2} 2 s^{2} S_{1 / 2}$ dipole-forbidden transition. They use high-resolution spectroscopy of a low-temperature tokamak plasma to obtain an energy of $3090.25 \pm 0.12 \mathrm{eV}$ for the upper level, which is in remarkable agreement with the HFR1 value of $3090.1 \mathrm{eV}$ in Table 1 . For Be-like Mg and Si the agreement between HFR1 and NIST is well within $2 \mathrm{eV}$.

Theoretical K-vacancy level energies for ions with $18 \leq Z \leq$ 26 and $6 \leq N \leq 9$ are listed by Safronova \& Shlyaptseva (1999), which allow a comparison with HFR1 for species with $18 \leq Z \leq$ 20. It is found that energy differences are within $3 \mathrm{eV}$ except for the eight levels in both Ar and Ca shown in Table 2. They mainly belong to the O-like type, and the HFR1 values are always larger by as much as $6 \mathrm{eV}$.

\subsection{Wavelengths}

Since theoretical wavelengths do not reach spectroscopic accuracy, statistical comparisons with measurements are essential inasmuch as they enable small empirical adjustments to make the computed values astrophysically useful. It is worth pointing out that, due to the lack of experimental data for ionic species of the second row, this procedure is only currently possible for systems with electron number $N \leq 9$.

Experimental wavelengths for Ar ions with $3 \leq N \leq 9$ have been reported by Biémont et al. (2000) as well as theoretical

TABLE 2

K-VACancy Level Energies in Ar and Ca Ions

\begin{tabular}{|c|c|c|c|c|c|}
\hline \multirow[b]{2}{*}{$N$} & \multirow[b]{2}{*}{ Level } & \multicolumn{2}{|c|}{$Z=18$} & \multicolumn{2}{|c|}{$Z=20$} \\
\hline & & $\begin{array}{c}\text { HFR1 } \\
(\mathrm{eV})\end{array}$ & $\begin{array}{l}\mathrm{SS}^{\mathrm{a}} \\
(\mathrm{eV})\end{array}$ & $\begin{array}{c}\text { HFR1 } \\
(\mathrm{eV})\end{array}$ & $\begin{array}{l}\mathrm{SS}^{\mathrm{a}} \\
(\mathrm{eV})\end{array}$ \\
\hline $6 \ldots \ldots \ldots \ldots \ldots . .$. & $1 s 2 p^{5}{ }^{1} P_{1}^{o}$ & 3165.4 & 3162.0 & 3935.5 & 3932.0 \\
\hline 7......................... & $1 s 2 p^{6}{ }^{2} S_{1 / 2}$ & 3157.8 & 3154.1 & 3925.7 & 3921.9 \\
\hline 8 & $1 s 2 s^{2} 2 p^{5}{ }^{3} P_{2}^{o}$ & 2992.3 & 2988.4 & 3734.8 & 3730.6 \\
\hline 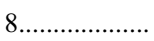 & $1 s 2 s^{2} 2 p^{5}{ }^{3} P_{1}^{o}$ & 2994.0 & 2990.4 & 3737.5 & 3733.8 \\
\hline 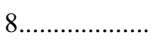 & $1 s 2 s^{2} 2 p^{5}{ }^{3} P_{0}^{o}$ & 2995.2 & 2991.9 & 3739.4 & 3736.3 \\
\hline 8......................... & $1 s 2 s^{2} 2 p^{5}{ }^{1} P_{1}^{o}$ & 3004.7 & 3000.5 & 3749.9 & 3745.4 \\
\hline 8........................ & $1 s 2 s 2 p^{6}{ }^{3} S_{1}$ & 3059.8 & 3054.6 & 3813.3 & 3808.0 \\
\hline 8 & $1 s 2 s 2 p^{6}{ }^{1} S_{0}$ & 3076.9 & 3071.2 & 3832.9 & 3827.3 \\
\hline
\end{tabular}

a Safronova \& Shlyaptseva (1999).

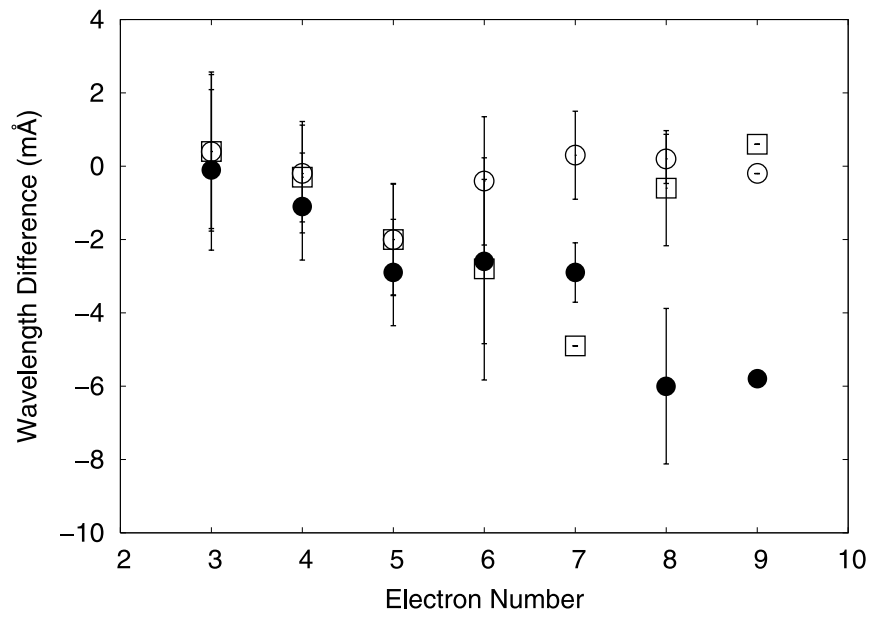

FIG. 2.- Average wavelength difference $\overline{\Delta \lambda_{e}}(\mathrm{~m} \AA)$ as a function of the electron number for ions in the $\mathrm{Ar}$ isonuclear sequence. $\overline{\Delta \lambda_{e}}$ is determined with respect to the spectroscopic values of Biémont et al. (2000). Filled circles: HFR1 values. Open circles: HFR2 results by Biémont et al. (2000). Squares: MCDF1 results by Biémont et al. (2000). A large error bar for the MCDF1 value at $N=7$ has been removed for clarity.

values computed with the HFR and YODA codes. The two latter data sets are to be hereafter referred to as HFR2 and MCDF1, respectively. As shown in Figure 2, a statistical comparison with the measured values can be carried out where the average theoryexperiment wavelength difference, $\overline{\Delta \lambda}_{e}$, is plotted as a function of the ion electron number $N$. It may be seen that theoretical wavelengths appear to be always shorter than the measured values. In the case of our HFR1 data, $\left|\overline{\Delta \lambda}_{e}\right|$ increases with $N$ from under $1 \mathrm{~m} \AA$ for $N=3$ to around $6 \mathrm{~m} \AA$ for $8 \leq N \leq 9$. A similar behavior is displayed by the MCDF1 values except for $8 \leq N \leq$ 9, where $\left|\overline{\Delta \lambda_{e}}\right|<1 \mathrm{~m} \AA$; this seems to indicate that the transition energies in these two ions have been adjusted with the experimental values. This is certainly also the case with the HFR 2 results for $3 \leq N \leq 9$ where $\left|\Delta \lambda_{e}\right|<1 \mathrm{~m} \AA$. However, as shown in Figure 2 , the standard deviations for all three theoretical data sets are around 2-3 mÅ which gives an indication of the level of accuracy that can be attained theoretically.

For the computed MCDF wavelengths listed by Chen \& Crasemann (1988) and Chen et al. (1997) for B- and C-like Ar, $\left|\overline{\Delta \lambda_{e}}\right|=2 \pm 2 \mathrm{~m} \AA$ and $\left|\overline{\Delta \lambda_{e}}\right|=4 \pm 6 \mathrm{~m} \AA$, respectively. The somewhat large standard deviation in the $\mathrm{C}$-like system is caused by transitions involving heavily mixed $\mathrm{K}$-vacancy states, in particular $1 s\left({ }^{2} S\right) 2 s 2 p^{4}\left({ }^{2} D\right){ }^{1} D_{2}$, but the level of agreement is in general similar to that discussed above for HFR1.

Differences between the HFR1 wavelengths and the measurements listed by Faenov et al. (1994) for ions with $12 \leq Z \leq 16$ and $4 \leq N \leq 9$ display a similar behavior to that found in the $\mathrm{Ar}$ ions; for each isonuclear sequence, $\left|\overline{\Delta \lambda_{e}}\right|$ increases with $N$. As expected, it also decreases with $Z$ along the isoelectronic sequence; for instance, in the case of $N=9,\left|\overline{\Delta \lambda_{e}}\right| \approx 18 \mathrm{~m} \AA$ for $Z=14$, while $\left|\overline{\Delta \lambda_{e}}\right| \approx 10 \mathrm{~m} \AA$ for $Z=16$. On the other hand, we have found three transitions in N-like ions $(N=7)$ with unusually large wavelength differences which are listed in Table 3. As a result of the comparisons with the spectroscopic data, the HFR1 wavelengths for systems with $3 \leq N \leq 9$ have been empirically shifted with $-\overline{\Delta \lambda_{e}}$.

Behar \& Netzer (2002) list wavelengths computed with the HULLAC code for $68 \mathrm{~K}$-transitions arising from the ground level of ions with $2 \leq N \leq 9$. In general, the agreement with HFR 1 is better than $10 \mathrm{~m} \AA$ and progressively improves with $Z$ up to $3 \mathrm{~m} \AA$ for $Z=20$. As depicted in Table 4 , there are, however, 
TABLE 3

HFR1 and Experimental Wavelengths with Large Differences

\begin{tabular}{ccccccc}
\hline \hline$Z$ & $N$ & Upper Level & Lower Level & $\begin{array}{c}\text { HFR1 } \\
(\AA)\end{array}$ & $\begin{array}{c}\text { Expt }^{\mathrm{a}} \\
(\AA)\end{array}$ & $\begin{array}{c}\mathrm{MZ}^{\mathrm{a}} \\
(\AA)\end{array}$ \\
\hline $14 \ldots \ldots \ldots \ldots \ldots \ldots \ldots$ & 7 & $1 s 2 p^{6}{ }^{2} S_{1 / 2}$ & $1 s^{2} 2 s^{2} 2 p^{3}{ }^{2} P_{3 / 2}^{o}$ & 6.6590 & 7.0156 & 7.0161 \\
$16 \ldots \ldots \ldots \ldots \ldots \ldots \ldots \ldots$ & 7 & $1 s\left({ }^{2} S\right) 2 s 2 p^{5}\left({ }^{3} P^{o}\right){ }^{4} P_{1 / 2}^{o}$ & $1 s^{2} 2 s 2 p^{4}{ }^{2} D_{3 / 2}$ & 5.3141 & 5.2804 & 5.2797 \\
& 7 & $1 s 2 p^{6}{ }^{2} S_{1 / 2}$ & $1 s^{2} 2 s^{2} 2 p^{3}{ }^{2} P_{3 / 2}^{o}$ & 5.0434 & 5.2863 & 5.2864 \\
\hline
\end{tabular}

${ }^{\text {a }}$ Experiment and calculation (MZ code) by Faenov et al. (1994).

several transitions with noticeable differences, specially for ions with $Z \leq 14$ and $6 \leq N \leq 9$, where the longer HULLAC wavelengths are probably more accurate than HFR1. Surprisingly large differences $(10 \mathrm{~m} \AA)$ are found between HULLAC and measurements for transitions in Li-like $\mathrm{Ne}$ for which the measured values agree with HFR1 to within $3 \mathrm{~m} \AA$ (see Table 4).

HFR1 $\mathrm{K} \alpha, \mathrm{K} \beta$, and edge transition energies for singly ionized species with $10 \leq Z \leq 20$ are compared in Table 5 with the compilation by Deslattes et al. (2003) of experimental and theoretical $\mathrm{X}$-ray transition energies. The HFR1 edge transition energy is estimated using the experimental ionization potential of the neutral and the HFR1 position for the lowest K-vacancy level of the singly ionized stage. The agreement with their theoretical and the experimental $\mathrm{K} \alpha$ transition energies is within $1.5 \mathrm{eV}$, but discrepancies as large as $7 \mathrm{eV}$ are found with the experimental $\mathrm{K} \beta$ values and with the edge energies $(\sim 10 \mathrm{eV})$ for $12 \leq Z \leq 16$. The soundness of this comparison may be limited by experimental difficulties in assigning weak features and by solid-state effects.

\subsection{A-Values}

Present $A$-values are computed with approximations AS1, AS2, and HFR1. As mentioned in $\S 2$, in AS2 and HFR1 nonorthogonal orbital bases are used which account for orbital relaxation in the radiative decay process. It has been found that the $1 \mathrm{~s}$ orbital is particularly different in valence and K-vacancy states. Since orthogonal bases are used in AS1, a comparison of AS1 and AS2 would give an indication of the importance of this effect.

The radiative data listed by Behar \& Netzer (2002) for K transitions, namely $f$ - and $A$-values, are consistently $10 \% \pm 5 \%$ below HFR1. To study this situation, we first look at the absorption $f$-value for the $1 s^{2}{ }^{1} S_{0} \rightarrow 1 s 2 p^{1} P_{1}^{o}$ resonance transition along the He isoelectronic sequence (see Fig. 3). It may be seen that the $f$-values by Behar \& Netzer are about $6 \%$ below those by Drake (1979), which are usually taken as the reference. On the other hand, HFR1 is 9\% higher and so is AS2 (6\%). As shown in Figure 3, if the HFR $1 f$-values are recomputed in a singleconfiguration approximation, the differences with Drake are reduced to $6 \%$ and AS1 also does somewhat better (3\%). The latter result suggests that perhaps taking into account core relaxation effects does not necessarily lead to improved radiative data. This hypothesis is confirmed by a comparison of available lifetime measurements in He- and Li-like ions with AS1 and AS2 (see Table 6). The computed lifetimes for these $n=2$ levels are sensitive to the wave functions, because most of them involve optically forbidden decay channels. Although the agreement of both $\mathrm{AS} 1$ and AS2 with experiment is within approximately $10 \%$, it is not clear which is the most accurate. As a conclusion, we are only confident that the accuracy of the HFR $1 A$-values is at around the $15 \%$ level.

This suggested level of accuracy is further supported by a comparison of the HFR $1 A$-values greater than $10^{13} \mathrm{~s}^{-1}$ in Ar ions with those computed by Biémont et al. (2000) in the HFR2 and MCDF1 approximations. In spite of their fine tuning of the transition matrix elements with the experimental energy levels in HFR2, the general agreement is well within $15 \%$ except for the few problematic transitions listed in Table 7 . It may be seen that for transitions in species with $N=5$ and $N=7$, HFR1 and HFR2 are in reasonable agreement in contrast to MCDF1. For $N=6$, the scatter is large which is surely due to strong admixture of the upper K-vacancy levels produced by the spin-orbit interaction.

$A$-values calculated by Chen \& Crasemann (1988) for B-like Ar using the MCDF method agree within $20 \%$ with HFR1, although on average they are $\sim 10 \%$ smaller. However, we find that those computed for C-like Ar by Chen et al. (1997), as shown in

TABLE 4

HFR1 and HULLAC Wavelengths with LaRge Differences

\begin{tabular}{|c|c|c|c|c|c|c|}
\hline$Z$ & $N$ & Upper level & Lower level & $\begin{array}{l}\text { HFR1 } \\
(\AA ̊)\end{array}$ & $\begin{array}{c}\text { HULLAC }^{\mathrm{a}} \\
(\AA)\end{array}$ & $\begin{array}{l}\text { Expt } \\
(\AA)\end{array}$ \\
\hline \multirow[t]{6}{*}{$10 \ldots \ldots \ldots \ldots \ldots \ldots \ldots \ldots$} & 3 & $1 s 2 s 2 p^{2} P_{1 / 2}^{o}$ & $1 s^{2} 2 s^{2} S_{1 / 2}$ & 13.658 & 13.648 & $13.655(3)^{\mathrm{b}}$ \\
\hline & 3 & $1 s 2 s 2 p^{2} P_{3 / 2}^{o}$ & $1 s^{2} 2 s^{2} S_{1 / 2}$ & 13.656 & 13.646 & $13.655(3)^{\mathrm{b}}$ \\
\hline & 4 & $1 s 2 s^{2} 2 p^{1} P_{1}^{o}$ & $1 s^{2} 2 s^{2}{ }^{1} S_{0}$ & 13.824 & 13.814 & \\
\hline & 6 & $1 s 2 s^{2} 2 p^{3}{ }^{3} D_{1}^{o}$ & $1 s^{2} 2 s^{2} 2 p^{2}{ }^{3} P_{0}$ & 14.225 & 14.239 & \\
\hline & 8 & $1 s 2 s^{2} 2 p^{5}{ }^{3} P_{2}^{o}$ & $1 s^{2} 2 s^{2} 2 p^{4}{ }^{3} P_{2}$ & 14.490 & 14.526 & \\
\hline & 9 & $1 s 2 s^{2} 2 p^{6}{ }^{2} S_{1 / 2}$ & $1 s^{2} 2 s^{2} 2 p^{5}{ }^{2} P_{3 / 2}^{o}$ & 14.596 & 14.631 & \\
\hline \multirow[t]{3}{*}{$12 \ldots \ldots \ldots \ldots \ldots \ldots \ldots \ldots \ldots$} & 6 & $1 s 2 s^{2} 2 p^{3}{ }^{3} D_{1}^{o}$ & $1 s^{2} 2 s^{2} 2 p^{2}{ }^{3} P_{0}^{3 / 2}$ & 9.622 & 9.631 & \\
\hline & 8 & $1 s 2 s^{2} 2 p^{5}{ }^{3} P_{2}^{o}$ & $1 s^{2} 2 s^{2} 2 p^{4}{ }^{3} P_{2}$ & 9.797 & 9.816 & \\
\hline & 9 & $1 s 2 s^{2} 2 p^{6}{ }^{2} S_{1 / 2}$ & $1 s^{2} 2 s^{2} 2 p^{5}{ }^{2} P_{3 / 2}^{o}$ & 9.875 & 9.895 & $9.9129^{c}$ \\
\hline \multirow[t]{2}{*}{ 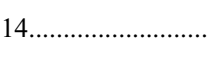 } & 8 & $1 s 2 s^{2} 2 p^{5}{ }^{3} P_{2}^{o}$ & $1 s^{2} 2 s^{2} 2 p^{4}{ }^{3} P_{2}$ & 7.052 & 7.063 & $7.0665^{\mathrm{c}}$ \\
\hline & 9 & $1 s 2 s^{2} 2 p^{6}{ }^{2} S_{1 / 2}$ & $1 s^{2} 2 s^{2} 2 p^{5}{ }^{2} P_{3 / 2}^{o}$ & 7.107 & 7.119 & $7.1244^{\mathrm{c}}$ \\
\hline
\end{tabular}

\footnotetext{
a Behar \& Netzer (2002)

b NIST database ver. 3.1.2 (Ralchenko et al. 2007) and Kramida \& Buchet-Poulizac (2006).

c Faenov et al. (1994) .
} 
TABLE 5

Transition ENERgies

\begin{tabular}{|c|c|c|c|c|c|}
\hline$Z$ & $\begin{array}{l}\mathrm{K} \alpha_{1} \\
(\mathrm{eV})\end{array}$ & $\begin{array}{l}\mathrm{K} \alpha_{2} \\
(\mathrm{eV})\end{array}$ & $\begin{array}{l}\mathrm{K} \beta_{1} \\
(\mathrm{eV})\end{array}$ & $\begin{array}{l}\mathrm{K} \beta_{3} \\
(\mathrm{eV})\end{array}$ & $\begin{array}{l}\text { Edge } \\
(\mathrm{eV})\end{array}$ \\
\hline \multirow[t]{3}{*}{$10 \ldots$} & 849.5 & 849.4 & & & 871.0 \\
\hline & 849.2 & 849.1 & & & 870.7 \\
\hline & $848.61(26)$ & $848.61(26)$ & $857.89(44)$ & $857.89(44)$ & $870.23(18)$ \\
\hline \multirow[t]{3}{*}{$12 \ldots \ldots \ldots$} & 1254.6 & 1254.6 & 1308.4 & 1308.4 & 1311.5 \\
\hline & 1254.4 & 1254.1 & & & 1312.3 \\
\hline & $1253.688(11)$ & $1253.437(13)$ & $1302.20(40)$ & $1302.20(40)$ & $1303.33(27)$ \\
\hline \multirow[t]{3}{*}{14} & 1741.2 & 1741.2 & 1841.8 & 1842.8 & 1849.2 \\
\hline & 1741.2 & 1739.7 & & 1841.8 & 1850.3 \\
\hline & $1739.985(19)$ & $1739.394(34)$ & $1835.96(40)$ & $1835.96(40)$ & $1839.13(37)$ \\
\hline \multirow[t]{3}{*}{16} & 2309.2 & 2306.8 & 2468.7 & 2470.2 & 2481.9 \\
\hline & 2308.8 & 2307.0 & 2467.5 & 2469.7 & 2481.7 \\
\hline & $2307.885(34)$ & $2306.700(38)$ & $2464.07(14)$ & & $2471.63(70)$ \\
\hline \multirow[t]{3}{*}{18} & 2958.7 & 2956.6 & 3192.7 & 3192.5 & 3208.4 \\
\hline & 2957.9 & 2955.9 & 3191.5 & 3191.3 & 3207.4 \\
\hline & $2957.682(16)$ & $2955.566(16)$ & $3190.49(24)$ & $3190.49(24)$ & $3206.14(54)$ \\
\hline \multirow[t]{3}{*}{$20 \ldots \ldots \ldots \ldots$} & 3692.4 & 3688.8 & 4015.3 & 4015.0 & 4049.9 \\
\hline & 3691.0 & 3687.6 & 4014.7 & 4014.3 & 4049.4 \\
\hline & $3691.719(49)$ & $3688.128(49)$ & $4012.76(38)$ & $4012.76(38)$ & $4050.48(30)$ \\
\hline
\end{tabular}

NoтE.-For each element, the first row lists HFR1 data, and the second and third rows respectively give computed and measured values by Deslattes et al. (2003).

Figure 4, are $37 \%$ higher. We also include in this plot the MCDF1 $A$-values by Biémont et al. (2000), which are within $15 \%$ of HFR1 once the problematic values of Table 7 are excluded. Moreover, there are several transitions by Chen et al. that show differences larger than a factor of 2 with HFR 1 which have not been taken into account in this comparison.

The comparison of the HFR1 $A$-values with those calculated by Faenov et al. (1994) using the MZ method for ions with $12 \leq Z \leq 16$ and $4 \leq N \leq 9$ shows a wide scatter; some transitions agree to better than $10 \%$, while large discrepancies are found for others.

\subsection{Radiative and Auger Widths}

Auger widths of K-vacancy levels are determined by including all the decay channels $C \epsilon l$, where $C$ are all the $n=2$ valence configurations of the $(N-1)$-electron daughter ion, and $l \leq 4$.

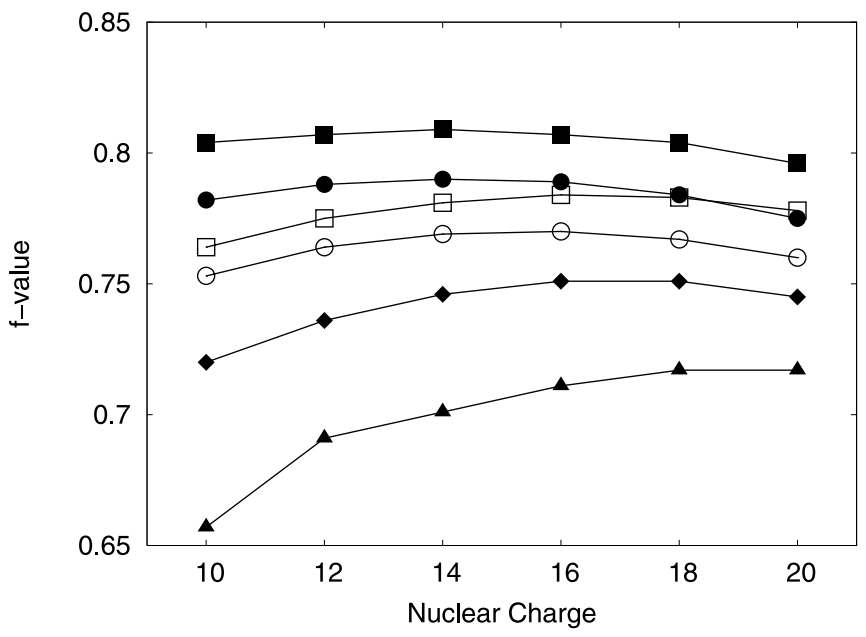

FIG. 3.- Absorption $f$-value for the $1 s^{2}{ }^{1} S_{0} \rightarrow 1 s 2 p{ }^{1} P_{1}^{o}$ resonance transition in the He isoelectronic sequence. Filled squares: HFR1. Filled circles: AS2. Open circles: AS1. Open squares: HFR, single configuration. Diamonds: Values by Drake (1979) calculated in a unified relativistic theory. Triangles: Data computed with the HULLAC code by Behar \& Netzer (2002).
TABLE 6

LIFETIMES FOR He- AND Li-LIKE IONS

\begin{tabular}{|c|c|c|c|c|c|}
\hline \multirow[b]{2}{*}{ Level } & \multirow[b]{2}{*}{$Z$} & \multirow[b]{2}{*}{$N$} & \multicolumn{3}{|r|}{$\tau(\mathrm{ns})$} \\
\hline & & & AS1 & AS2 & Expt \\
\hline \multirow[t]{4}{*}{$1 s 2 s^{3} S_{1} \ldots \ldots \ldots \ldots \ldots$} & 10 & 2 & $9.10+4$ & $1.01+5$ & $9.17(4)+4^{\mathrm{a}}$ \\
\hline & 12 & 2 & $1.37+4$ & $1.50+4$ & $1.361(49)+4^{\mathrm{b}}$ \\
\hline & 16 & 2 & $7.04+2$ & $7.55+2$ & $7.03(4)+2^{\mathrm{c}}$ \\
\hline & 18 & 2 & $2.11+2$ & $2.24+2$ & $2.03(12)+2^{\mathrm{d}}$ \\
\hline $1 s 2 p^{3} P_{0}^{o} \ldots \ldots \ldots \ldots$ & 18 & 2 & 5.11 & 4.60 & $4.87(44)^{\mathrm{e}}$ \\
\hline \multirow[t]{3}{*}{$1 s 2 p^{3} P_{1}^{o} \ldots \ldots \ldots \ldots$} & 12 & 2 & $3.26-2$ & $3.07-2$ & $2.90(15)-2^{f}$ \\
\hline & 14 & 2 & $7.08-3$ & $6.73-3$ & $6.45(30)-3^{\mathrm{f}}, 6.35(33)-3^{\mathrm{g}}$ \\
\hline & 16 & 2 & $1.93-3$ & $1.85-3$ & $1.57(18)-3^{\mathrm{g}}$ \\
\hline \multirow[t]{2}{*}{$1 s 2 p^{3} P_{2}^{o} \ldots \ldots \ldots \ldots$} & 16 & 2 & 2.80 & 2.60 & $2.5(2)^{\mathrm{h}}$ \\
\hline & 18 & 2 & 1.58 & 1.50 & $1.62(8)^{\mathrm{e}}$ \\
\hline $1 s 2 p^{1} P_{1}^{o} \ldots \ldots \ldots \ldots$ & 14 & 2 & $2.59-5$ & $2.52-5$ & $2.9(10)-5^{\mathrm{i}}$ \\
\hline \multirow[t]{4}{*}{$1 s 2 s 2 p{ }^{4} P_{5 / 2}^{o} \ldots \ldots$} & 10 & 3 & $1.09+1$ & $1.01+1$ & $1.04(15)+1^{j}$ \\
\hline & 14 & 3 & 2.19 & 2.07 & $2.1(1)^{\mathrm{k}}$ \\
\hline & 16 & 3 & 1.14 & 1.09 & $1.1(2)^{\mathrm{h}}$ \\
\hline & 18 & 3 & $6.33-1$ & $6.08-1$ & $5.94(16)-1^{1}$ \\
\hline \multirow[t]{2}{*}{$1 s 2 p^{2}{ }^{4} P_{1 / 2} \ldots \ldots \ldots$} & 10 & 3 & $5.46-1$ & $4.75-1$ & $5.3(5)-1^{\mathrm{m}}$ \\
\hline & 12 & 3 & $1.56-1$ & $1.40-1$ & $1.74(17)-1^{\mathrm{n}}$ \\
\hline \multirow[t]{2}{*}{$1 s 2 p^{2}{ }^{4} P_{3 / 2} \ldots \ldots \ldots$} & 10 & 3 & $3.90-1$ & $3.46-1$ & $4.0(4)-1^{\mathrm{m}}$ \\
\hline & 12 & 3 & $9.38-2$ & $8.35-2$ & $9.4(12)-2^{\mathrm{n}}$ \\
\hline $1 s 2 p^{2}{ }^{4} P_{5 / 2} \ldots \ldots \ldots$ & 12 & 3 & $8.80-3$ & $7.45-3$ & $9(4)-3^{n}$ \\
\hline
\end{tabular}

Note.-The format for the fourth, fifth, and sixth columns is $a \pm b \equiv a \times 10^{ \pm b}$.

${ }^{a}$ Value from Träbert et al. (1999).

b Value from Stefanelli et al. (1995).

${ }^{c}$ Value from Crespo López-Urrutia et al. (2006).

${ }^{\mathrm{d}}$ Value from Hubricht \& Träbert (1987).

e Value from Davis \& Marrus (1977).

${ }^{\mathrm{f}}$ Value from Armour et al. (1981).

g Value from Varghese et al. (1976a).

${ }^{\text {h }}$ Value from Cocke et al. (1974).

${ }^{i}$ Value from Varghese et al. (1976b).

j Value from Groeneveld et al. (1975).

${ }^{\mathrm{k}}$ Value from Haselton et al. (1975).

${ }^{1}$ Value from Dohmann \& Mann (1979).

${ }^{m}$ Value from Knystautas \& Druetta (1985).

${ }^{\mathrm{n}}$ Value from Hellmann \& Träbert (1985). 
TABLE 7

Transitions in Ar Ions with Questionable $A$-Values

\begin{tabular}{|c|c|c|c|c|c|}
\hline$N$ & Upper Level & Lower Level & $\begin{array}{r}\text { HFR1 } \\
\left(\mathrm{s}^{-1}\right)\end{array}$ & $\begin{array}{c}\mathrm{HFR}^{\mathrm{a}} \\
\left(\mathrm{s}^{-1}\right)\end{array}$ & $\begin{array}{c}\mathrm{MCDF}^{\mathrm{a}} 1^{-} \\
\left(\mathrm{s}^{-1}\right)\end{array}$ \\
\hline \multirow[t]{4}{*}{5} & $1 s\left({ }^{2} S\right) 2 s 2 p^{3}\left({ }^{3} D^{o}\right){ }^{4} D_{3 / 2}^{o}$ & $1 s^{2} 2 s 2 p^{2}{ }^{4} P_{3 / 2}$ & $1.49+13$ & $1.48+13$ & $1.08+13$ \\
\hline & $1 s\left({ }^{2} S\right) 2 s 2 p^{3}\left({ }^{1} D^{o}\right)^{2} D_{3 / 2}^{o}$ & $1 s^{2} 2 s 2 p^{2}{ }^{2} D_{5 / 2}$ & $2.36+13$ & $2.20+13$ & $1.77+13$ \\
\hline & $1 s\left({ }^{2} S\right) 2 s 2 p^{3}\left({ }^{1} P^{o}\right)^{2} P_{1 / 2}^{o}$ & $1 s^{2} 2 s 2 p^{2}{ }^{2} S_{1 / 2}$ & $2.62+13$ & $2.97+13$ & $1.72+13$ \\
\hline & $1 s 2 p^{4} D_{3 / 2}$ & $1 s^{2} 2 p^{3}{ }^{T} P_{1 / 2}^{o}$ & $1.02+13$ & $9.00+12$ & $6.80+12$ \\
\hline \multirow[t]{12}{*}{ 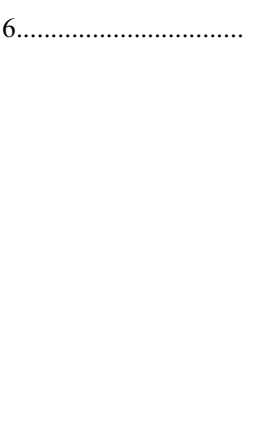 } & $1 s 2 s^{2} 2 p^{33} S_{1}^{o}$ & $1 s^{2} 2 s^{2} 2 p^{2}{ }^{1 / 2} P_{2}$ & $3.11+13$ & $2.33+13$ & $3.50+13$ \\
\hline & $1 s\left({ }^{2} S\right) 2 s 2 p^{4}\left({ }^{2} D\right){ }^{3} D_{2}$ & $1 s^{2} 2 s 2 p^{3}{ }^{3} D_{2}^{o}$ & $7.26+13$ & $1.28+13$ & $2.00+11$ \\
\hline & $1 s\left({ }^{2} S\right) 2 s 2 p^{4}\left({ }^{2} D\right){ }^{3} D_{2}$ & $1 s^{2} 2 s 2 p^{3}{ }^{3} D_{3}^{o}$ & $3.45+13$ & $6.29+13$ & $9.60+13$ \\
\hline & $1 s\left({ }^{2} S\right) 2 s 2 p^{4}\left({ }^{2} D\right){ }^{3} D_{2}$ & $1 s^{2} 2 s 2 p^{3}{ }^{3} P_{1}^{o}$ & $2.66+11$ & $3.52+13$ & $3.51+13$ \\
\hline & $1 s\left({ }^{2} S\right) 2 s 2 p^{4}\left({ }^{2} D\right){ }^{3} D_{2}$ & $1 s^{2} 2 s 2 p^{3}{ }^{3} P_{2}^{o}$ & $3.29+13$ & $4.70+12$ & $1.74+13$ \\
\hline & $1 s\left({ }^{2} S\right) 2 s 2 p^{4}\left({ }^{2} D\right){ }^{3} D_{1}$ & $1 s^{2} 2 s 2 p^{3}{ }^{3} P_{2}^{o}$ & $8.07+12$ & $1.12+13$ & $5.80+12$ \\
\hline & $1 s\left({ }^{2} S\right) 2 s 2 p^{4}\left({ }^{4} P\right){ }^{3} P_{2}$ & $1 s^{2} 2 s 2 p^{3}{ }^{3} D_{3}^{o}$ & $6.94+13$ & $4.58+13$ & $1.50+13$ \\
\hline & $1 s\left({ }^{2} S\right) 2 s 2 p^{4}\left({ }^{4} P\right){ }^{3} P_{2}$ & $1 s^{2} 2 s 2 p^{3}{ }^{3} P_{2}^{o}$ & $7.57+12$ & $3.89+13$ & $2.50+13$ \\
\hline & $1 s\left({ }^{2} S\right) 2 s 2 p^{4}\left({ }^{2} D\right){ }^{1} D_{2}$ & $1 s^{2} 2 s 2 p^{3}{ }^{1} P_{1}^{o}$ & $1.01+13$ & $1.45+13$ & $1.79+13$ \\
\hline & $1 s\left({ }^{2} S\right) 2 s 2 p^{4}\left({ }^{2} P\right){ }^{3} P_{2}$ & $1 s^{2} 2 s 2 p^{3}{ }^{1} D_{2}^{o}$ & $2.23+13$ & $1.39+13$ & $3.94+13$ \\
\hline & $1 s\left({ }^{2} S\right) 2 s 2 p^{4}\left({ }^{2} P\right){ }^{3} P_{2}$ & $1 s^{2} 2 s 2 p^{3}{ }^{3} S_{1}^{o}$ & $2.39+13$ & $3.22+13$ & $1.53+13$ \\
\hline & $1 s\left({ }^{2} S\right) 2 s 2 p^{4}\left({ }^{2} P\right){ }^{3} P_{2}$ & $1 s^{2} 2 s 2 p^{3}{ }^{1} P_{1}^{o}$ & $1.29+13$ & $9.30+12$ & $5.50+12$ \\
\hline \multirow[t]{3}{*}{ 7... } & $1 s\left({ }^{2} S\right) 2 s 2 p^{5}\left({ }^{3} P^{o}\right)^{2} P_{3 / 2}^{o}$ & $1 s^{2} 2 s 2 p^{42} S_{1 / 2}$ & $2.67+13$ & $2.72+13$ & $5.30+12$ \\
\hline & $1 s\left({ }^{2} S\right) 2 s 2 p^{5}\left({ }^{3} P^{o}\right)^{2} P_{1 / 2}^{o}$ & $1 s^{2} 2 s 2 p^{4}{ }^{2} D_{3 / 2}$ & $1.39+14$ & $1.41+14$ & $2.94+13$ \\
\hline & $1 s\left({ }^{2} S\right) 2 s 2 p^{5}\left({ }^{1} P^{o}\right)^{2} P_{1 / 2}^{o}$ & $1 s^{2} 2 s 2 p^{4}{ }^{2} P_{3 / 2}$ & $2.58+13$ & $2.61+13$ & $1.35+14$ \\
\hline
\end{tabular}

Note.-The format for the fourth, fifth, and sixth columns is $a \pm b \equiv a \times 10^{ \pm b}$.

${ }^{\mathrm{a}}$ HFR2 and MCDF1 results computed by Biémont et al. (2000).

Biémont et al. (2000) have computed with HFR and the MCDF YODA code Auger widths for K-vacancy states in Ar ions with $3 \leq N \leq 9$ (data sets referred to as HFR2 and MCDF1, respectively). A reasonable agreement (within 20\%) with HFR1 is found except for the two levels in the Li-like system $(N=3)$ and the five levels in the Be-like system $(N=4)$ shown in Table 8 . The MCDF1 K-vacancy levels in the N-like ion $(N=7)$ are also on average around $40 \%$ larger. The discrepant HFR2 results for the Li-like ion are attributed to typos, since a recalculation results in data very similar to the HFR 1 values.

MCDF radiative and Auger widths have also been computed for Be-, B-, and C-like Ar by Chen (1985), Chen \& Crasemann (1988), and Chen et al. (1997), respectively. For the Be- and Blike systems, they agree with HFR 1 to within $10 \%$ except for the small Auger widths $\left(A_{a}<10^{12} \mathrm{~s}^{-1}\right)$ of the $1 s 2 s 2 p^{2}{ }^{5} P_{j}$ levels in Be-like Ar. For the C-like system, on the other hand, radiative

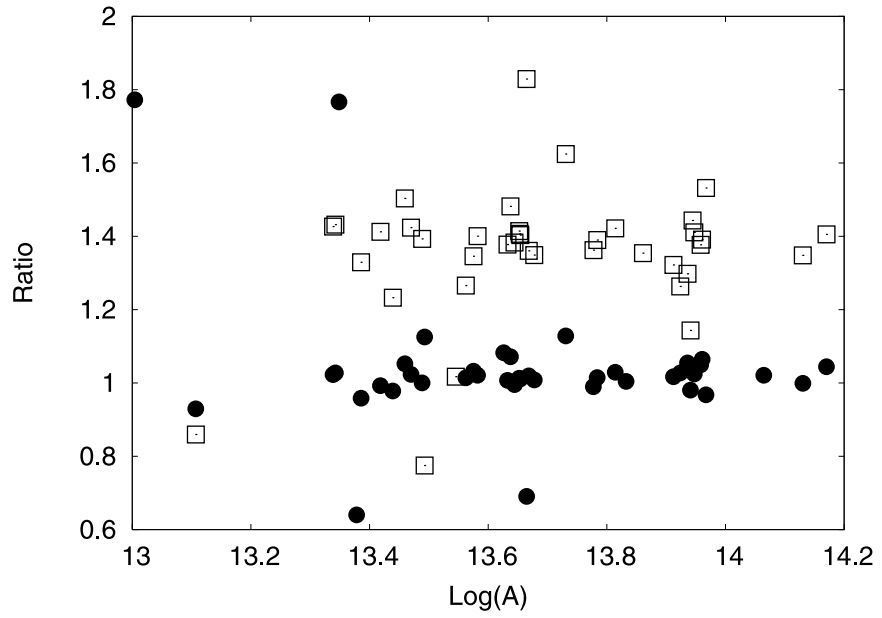

FIG. 4.-Comparison of the present HFR $1 A$-values for K transitions in C-like Ar with two independent MCDF calculations. Squares: Chen et al. (1997). Circles: MCDF1 $A$-values by Biémont et al. (2000). It is found that the data by Chen et al. (1997) are on average larger by $37 \%$ with respect to HFR 1 . and Auger widths by Chen et al. are on average larger than HFR 1 by $40 \%$ and $30 \%$, respectively, with some differences being as large as a factor of 2. Such a poor accord can be appreciated in Figure 5, where the MCDF1 data by Biémont et al. (2000) is also shown. The latter agrees with HFR1 to within $20 \%$.

The total K-widths quoted by Behar \& Netzer (2002) follow a similar trend to that found for their $A$-values: on average they are found to be about $15 \%$ below HFR1, although for some levels large discrepancies are encountered (see Table 9). For such levels, it is shown that the agreement between HFR1 and AS1 is around $5 \%$.

The Auger rates for ions with atomic number $12 \leq Z \leq 16$ and electron number $4 \leq N \leq 9$ computed with the $\mathrm{MZ}$ code by Faenov et al. (1994) are found to be in complete disagreement with HFR1.

In their study of the fluorescence database by Kaastra \& Mewe (1993), Gorczyca et al. (2003) have computed with AUTOSTRUCTURE configuration-averaged fluorescence yields for the $\mathrm{K}$-vacancy configurations $1 s 2 s^{2} 2 p$ in Be-like ions and $1 s 2 s^{2} 2 p^{6}$ in F-like ions. They also list yields estimated from the MCDF data of Chen (1985) and from the widths computed with HULLAC

TABLE 8

Questionable Auger Widths for Ar Ions

\begin{tabular}{clcccc}
\hline \hline \multirow{2}{*}{$N$} & \multicolumn{1}{c}{ Level } & $\begin{array}{c}\text { HFR1 } \\
\left(\mathrm{s}^{-1}\right)\end{array}$ & $\begin{array}{c}\text { AS1 } \\
\left(\mathrm{s}^{-1}\right)\end{array}$ & $\begin{array}{c}\mathrm{HFR}^{\mathrm{a}} \\
\left(\mathrm{s}^{-1}\right)\end{array}$ & $\begin{array}{c}\mathrm{MCDF}^{\mathrm{a}} \\
\left(\mathrm{s}^{-1}\right)\end{array}$ \\
\hline $3 \ldots \ldots \ldots \ldots .$. & $1 s\left({ }^{2} S\right) 2 p^{2}\left({ }^{3} P\right){ }^{2} P_{1 / 2}$ & $1.34+11$ & $1.19+11$ & $1.70+12$ & $2.00+11$ \\
& $1 s\left({ }^{2} S\right) 2 p^{2}\left({ }^{(} S\right){ }^{2} S_{1 / 2}$ & $2.41+13$ & $2.15+13$ & $6.39+13$ & $2.21+13$ \\
$4 \ldots \ldots \ldots \ldots$. & $1 s 2 s^{2} 2 p{ }^{1} P_{1}^{o}$ & $1.14+14$ & $1.20+14$ & $5.69+13$ & $1.08+14$ \\
& $1 s\left({ }^{2} S\right) 2 s 2 p^{2}\left({ }^{4} P\right){ }^{3} P_{1}$ & $9.75+13$ & $9.62+13$ & $1.10+14$ & $5.23+13$ \\
& $1 s\left({ }^{2} S\right) 2 s 2 p^{2}\left({ }^{2} D\right){ }^{3} D_{1}$ & $8.63+13$ & $9.57+13$ & $7.53+13$ & $5.23+13$ \\
& $1 s\left({ }^{2} S\right) 2 s 2 p^{2}\left({ }^{4} P\right){ }^{3} P_{2}$ & $4.23+13$ & $3.73+13$ & $4.96+13$ & $5.41+13$ \\
& $1 s 2 p^{3}{ }^{3} S_{1}^{o}$ & $5.06+12$ & $6.23+12$ & $1.90+13$ & $4.50+12$
\end{tabular}

NoтE.-The format for the third, fourth, fifth, and sixth columns is $a \pm b \equiv a \times 10^{ \pm b}$.

${ }^{\mathrm{a}}$ HFR2 and MCDF1 results computed by Biémont et al. (2000). 


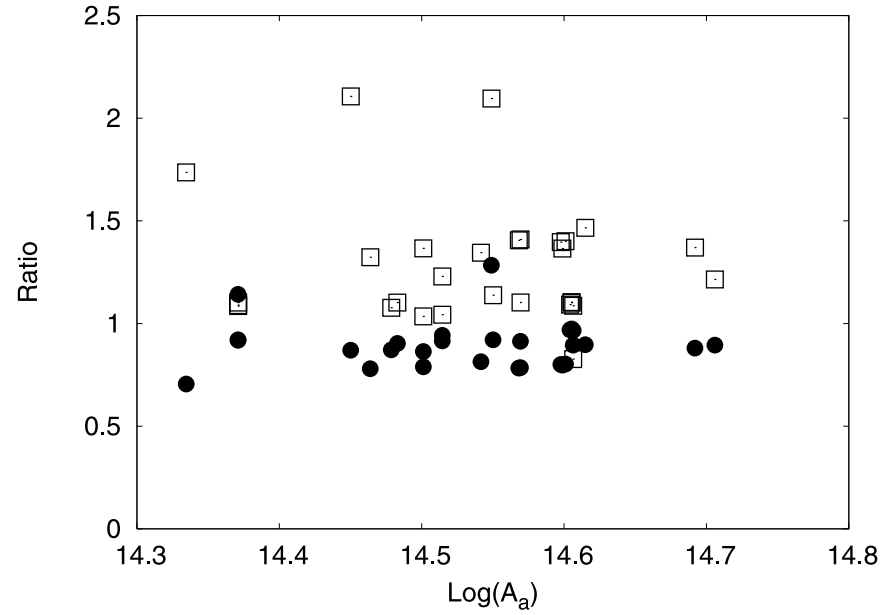

FIG. 5.-Comparison of the present HFR1 Auger widths for K-vacancy levels in C-like Ar with two independent MCDF calculations. Squares: Chen et al. (1997). Circles: MCDF1 Auger widths by Biémont et al. (2000). It is found that the data by Chen et al. (1997) are on average larger by $30 \%$.

by Behar \& Netzer (2002), the latter having been revised due to missing Auger channels and misquoted widths. In the comparison presented in Table 10, it may be seen that our HFR1 fluorescence yields agree with those by Gorczyca et al. (2003) to within $10 \%$ except for the Ne F-like ion (20\%). Differences may be due to the nonorthogonal orbital bases and neglect of twobody effects in the HFR code. The agreement between HFR 1 and Behar \& Netzer (2002) for the Be-like configuration is within 6\% but is poorer for the F-like (20\%), and that with Chen (1985) for the Be-like ion is better than $15 \%$.

\section{SUPPLEMENTARY ELECTRONIC TABLES}

Computed energy levels, wavelengths, $A$-values, $g f$-values, and radiative and Auger widths for ions in the $\mathrm{Ne}, \mathrm{Mg}, \mathrm{Si}, \mathrm{S}, \mathrm{Ar}$, and $\mathrm{Ca}$ isonuclear sequences can be accessed from Tables 11 and 12 in the electronic version of the Astrophysical Journal Supplement. The printed samples show data for ions of the Ne isonuclear with electron number $N \leq 3$. It may be seen that in Table 11 levels are identified with the vector $(Z, N, i, 2 S+1, L, 2 J$, Level),

TABLE 9

Questionable HULLAC K-VAcancy LeVel Widths

\begin{tabular}{|c|c|c|c|c|c|}
\hline$Z$ & $N$ & Level & $\begin{array}{c}\text { HFR1 } \\
\left(\mathrm{s}^{-1}\right)\end{array}$ & $\begin{array}{l}\text { AS1 } \\
\left(\mathrm{s}^{-1}\right)\end{array}$ & $\begin{array}{c}\text { HULLAC }^{\mathrm{a}} \\
\left(\mathrm{s}^{-1}\right)\end{array}$ \\
\hline \multirow[t]{4}{*}{$10 \ldots \ldots \ldots \ldots \ldots$} & 5 & $1 s 2 s^{2} 2 p^{2}{ }^{2} P_{1 / 2}$ & $1.03+14$ & $1.08+14$ & $3.55+13$ \\
\hline & 6 & $1 s 2 s^{2} 2 p^{3}{ }^{3} S_{1}^{o}$ & $1.06+14$ & $1.06+14$ & $5.76+13$ \\
\hline & 8 & $1 s 2 s^{2} 2 p^{5}{ }^{3} P_{2}^{o}$ & $3.71+14$ & $3.71+14$ & $2.55+14$ \\
\hline & 9 & $1 s 2 s^{2} 2 p^{6}{ }^{2} S_{1 / 2}$ & $3.92+14$ & $3.88+14$ & $5.80+12$ \\
\hline \multirow[t]{2}{*}{$12 \ldots \ldots \ldots$} & 5 & $1 s 2 s^{2} 2 p^{2}{ }^{2} P_{1 / 2}$ & $1.20+14$ & $1.33+14$ & $5.08+13$ \\
\hline & 6 & $1 s 2 s^{2} 2 p^{3}{ }^{3} S_{1}^{o}$ & $1.44+14$ & $1.48+14$ & $7.95+13$ \\
\hline \multirow[t]{2}{*}{14} & 5 & $1 s 2 s^{2} 2 p^{2}{ }^{2} P_{1 / 2}$ & $1.63+14$ & $1.67+14$ & $7.58+13$ \\
\hline & 6 & $1 s 2 s^{2} 2 p^{3}{ }^{3} S_{1}^{o}$ & $2.05+14$ & $2.23+14$ & $1.16+14$ \\
\hline \multirow[t]{2}{*}{$16 \ldots \ldots \ldots \ldots$} & 5 & $1 s 2 s^{2} 2 p^{2}{ }^{2} P_{1 / 2}$ & $2.11+14$ & $2.13+14$ & $1.14+14$ \\
\hline & 6 & $1 s 2 s^{2} 2 p^{3}{ }^{3} S_{1}^{o}$ & $2.80+14$ & $2.95+14$ & $1.70+14$ \\
\hline \multirow[t]{2}{*}{$18 \ldots \ldots \ldots \ldots \ldots \ldots \ldots$} & 5 & $1 s 2 s^{2} 2 p^{2}{ }^{2} P_{1 / 2}$ & $2.76+14$ & $2.76+14$ & $1.69+14$ \\
\hline & 6 & $1 s 2 s^{2} 2 p^{3}{ }^{3} S_{1}^{o}$ & $3.57+14$ & $3.63+14$ & $2.46+14$ \\
\hline \multirow[t]{2}{*}{$20 \ldots \ldots \ldots \ldots \ldots \ldots$} & 5 & $1 s 2 s^{2} 2 p^{2}{ }^{2} P_{1 / 2}$ & $3.61+14$ & $3.59+14$ & $2.47+14$ \\
\hline & 6 & $1 s 2 s^{2} 2 p^{3}{ }^{3} S_{1}^{o}$ & $4.51+14$ & $4.45+14$ & $3.46+14$ \\
\hline
\end{tabular}

Notes.-The level width is given by the sum of the radiative and Auger widths. In addition, the format of the fourth, fifth, and sixth columns is $a \pm b \equiv a \times 10^{ \pm b}$

a Widths computed with the HULLAC code by Behar \& Netzer (2002).
TABLE 10

Configuration-averaged Fluorescence Yields

\begin{tabular}{ccccccc}
\hline \hline \multirow{2}{*}{$N$} & $Z$ & Configuration & HFR1 & AS3 $^{\mathrm{a}}$ & HULLAC $^{\mathrm{b}}$ & MCDF $^{\mathrm{c}}$ \\
\hline $4 \ldots \ldots \ldots \ldots$. & 10 & $1 s 2 s^{2} 2 p$ & 0.0223 & 0.0201 & 0.0209 & 0.0191 \\
& 12 & & 0.0423 & 0.0393 & 0.0414 & 0.0377 \\
& 14 & & 0.0691 & 0.0658 & 0.0685 & \\
16 & & 0.1008 & 0.0974 & 0.0984 & \\
& 18 & & 0.1340 & 0.1309 & 0.1273 & 0.1237 \\
& 20 & & 0.1673 & 0.1646 & 0.1569 & \\
$10 \ldots \ldots \ldots \ldots$ & $1 s 2 s^{2} 2 p^{6}$ & 0.0185 & 0.0147 & 0.0215 & \\
& 12 & & 0.0332 & 0.0298 & 0.0380 & \\
& 14 & & 0.0564 & 0.0528 & 0.0630 & \\
& 16 & & 0.0895 & 0.0855 & 0.0983 & \\
18 & & 0.1343 & 0.1286 & 0.1443 & \\
& & & 0.1867 & 0.1818 & 0.2001 & \\
\hline
\end{tabular}

a Computed with AUTOSTRUCTURE by Gorczyca et al. (2003) including relativistic corrections.

b Estimated by Gorczyca et al. (2003) from the widths computed with HULLAC by Behar \& Netzer (2002) after some revision.

${ }^{c}$ Calculated by Gorczyca et al. (2003) from the MCDF data by Chen (1985) .

where $Z$ is the atomic number, $N$ is the electron number, $2 S+1$ is the spin multiplicity, $L$ is the total orbital angular momentum quantum number, $J$ is the total angular momentum quantum number, and Level is the level configuration assignment. For each level, the spectroscopic energy (when available), the computed HFR1 energy, and its radiative width $\left(A_{r}\right)$ are listed. For K-vacancy levels, the Auger width $\left(A_{a}\right)$ and fluorescence yield are additionally given. In Table 12 transitions are identified with the vector $(Z, N, k, i)$ where $k$ and $i$ are the upper and lower level indices, respectively, tabulating its computed wavelength, $A$-value, and $g f$-value.

\section{SUMMARY AND CONCLUSIONS}

Extensive data sets containing energy levels, wavelengths, $A$-values and radiative and Auger widths for K-vacancy levels in the $\mathrm{Ne}, \mathrm{Mg}, \mathrm{Si}, \mathrm{S}, \mathrm{Ar}$, and $\mathrm{Ca}$ isonuclear sequences have been computed with the atomic structure codes HFR and AUTOSTRUCTURE which include relativistic corrections. For ionic species of the second row with electron numbers $11 \leq N \leq 20$, it is the first time that such data become available. For first-row ions ( $3 \leq N \leq 10)$, detailed comparisons have been carried out with available measurements and theoretical values which have brought forth the consistency and accuracy of the present data sets.

Comparisons of the present HFR1 K-vacancy level energies with those listed in the NIST database and with recent measurements support an accuracy rating for ions with electron number $N \leq 4$ of better than $2 \mathrm{eV}$. Furthermore, we have shown that the NIST energies for K-vacancy levels in Li-like Ar are incorrect. Comparisons of the HFR1 wavelengths with the spectroscopic values reported for ions with $N \leq 9$ by Faenov et al. (1994) and Biémont et al. (2000) show that the theoretical wavelengths are consistently shorter. Moreover, along an isonuclear sequence, the average theory-experiment wavelength difference $\left|\overline{\Delta \lambda}_{e}\right|$ increases with $N$. This behavior enables empirical corrections to be made to the HFR1 wavelengths in ions with $N \leq 9$ that are bound to improve accuracy to around 2-3 mA. Because of a complete absence of spectroscopic measurements for $\mathrm{K}$ transitions in ions of the second row, this fine-tuning procedure cannot be extended at present to species with $N>9$ whose wavelengths therefore remain uncorrected.

The $A$-values and Auger widths for the Ar isonuclear sequence previously computed by Biémont et al. (2000) with HFR and the 
TABLE 11

Valence and K-Vacancy Levels for the Ne, Mg, Si, S, Ar, and Ca Isonuclear Sequences

\begin{tabular}{|c|c|c|c|c|c|c|c|c|c|c|c|}
\hline$Z$ & $N$ & $i$ & $2 S+1$ & $L$ & $2 J$ & Level & $\begin{array}{c}E(\mathrm{NIST}) \\
(\mathrm{eV})\end{array}$ & $\begin{array}{c}E(\text { HFR } 1) \\
(\mathrm{eV})\end{array}$ & $\begin{array}{l}A_{r}(i) \\
\left(\mathrm{s}^{-1}\right)\end{array}$ & $\begin{array}{l}A_{a}(i) \\
\left(\mathrm{s}^{-1}\right)\end{array}$ & Yield \\
\hline 10 & 1 & 1 & 2 & 0 & 1 & $1 \mathrm{~s} 2 \mathrm{~S} 1 / 2$ & 0.0000 & 0.0000 & & & \\
\hline 10 & 1 & 2 & 2 & 1 & 1 & $2 \mathrm{p} 2 \mathrm{P} 1 / 2$ & 1021.4970 & 1021.4975 & & & \\
\hline 10 & 1 & 3 & 2 & 0 & 1 & $2 \mathrm{~s} 2 \mathrm{~S} 1 / 2$ & 1021.5180 & 1021.5177 & & & \\
\hline 10 & 1 & 4 & 2 & 1 & 3 & $2 \mathrm{p} 2 \mathrm{P} 3 / 2$ & 1021.9530 & 1021.9528 & & & \\
\hline 10 & 2 & 1 & 1 & 0 & 0 & 1s2 1S0 & 0.0000 & 0.0000 & & & \\
\hline 10 & 2 & 2 & 3 & 0 & 2 & $1 \mathrm{~s} 2 \mathrm{~s} 3 \mathrm{~S} 1$ & 905.0775 & 904.4935 & & & \\
\hline 10 & 2 & 3 & 3 & 1 & 0 & $1 \mathrm{~s} 2 \mathrm{p} 3 \mathrm{Po} 0$ & 914.7805 & 914.1721 & $1.03 \mathrm{E}+08$ & & \\
\hline 10 & 2 & 4 & 3 & 1 & 2 & $1 \mathrm{~s} 2 \mathrm{p} 3 \mathrm{Po} 1$ & 914.8177 & 914.2734 & $3.78 \mathrm{E}+09$ & & \\
\hline 10 & 2 & 5 & 3 & 1 & 4 & $1 \mathrm{~s} 2 \mathrm{p} 3 \mathrm{Po} 2$ & 915.0099 & 914.4848 & $1.14 \mathrm{E}+08$ & & \\
\hline 10 & 2 & 6 & 1 & 0 & 0 & $1 \mathrm{~s} 2 \mathrm{~s} 1 \mathrm{~S} 0$ & 915.3360 & 915.2111 & $1.54 \mathrm{E}+02$ & & \\
\hline 10 & 2 & 7 & 1 & 1 & 2 & 1s2p 1Po1 & 922.0163 & 921.8277 & $9.88 \mathrm{E}+12$ & & \\
\hline 10 & 3 & 1 & 2 & 0 & 1 & $1 \mathrm{~s} 22 \mathrm{~s} 2 \mathrm{~S} 1 / 2$ & 0.0000 & 0.0000 & & & \\
\hline 10 & 3 & 2 & 2 & 1 & 1 & $1 \mathrm{~s} 22 \mathrm{p} 2 \mathrm{Po} 1 / 2$ & 15.8888 & 15.8681 & $5.50 \mathrm{E}+08$ & & \\
\hline 10 & 3 & 3 & 2 & 1 & 3 & $1 \mathrm{~s} 22 \mathrm{p} 2 \mathrm{Po} 3 / 2$ & 16.0933 & 16.0709 & $5.72 \mathrm{E}+08$ & & \\
\hline 10 & 3 & 4 & 2 & 0 & 1 & $1 \mathrm{~s} 2 \mathrm{~s} 22 \mathrm{~S} 1 / 2$ & & 891.0215 & $5.31 \mathrm{E}+11$ & $1.14 \mathrm{E}+14$ & 0.0046 \\
\hline 10 & 3 & 5 & 4 & 1 & 1 & $1 \mathrm{~s}(2 \mathrm{~S}) 2 \mathrm{~s} 2 \mathrm{p}(3 \mathrm{Po}) 4 \mathrm{Po} 1 / 2$ & & 895.1820 & $2.68 \mathrm{E}+08$ & $<5.00 \mathrm{E}+08$ & 1.0000 \\
\hline 10 & 3 & 6 & 4 & 1 & 3 & $1 \mathrm{~s}(2 \mathrm{~S}) 2 \mathrm{~s} 2 \mathrm{p}(3 \mathrm{Po}) 4 \mathrm{Po} 3 / 2$ & & 895.2770 & $6.86 \mathrm{E}+08$ & $1.00 \mathrm{E}+09$ & 0.4069 \\
\hline 10 & 3 & 7 & 4 & 1 & 5 & $1 \mathrm{~s}(2 \mathrm{~S}) 2 \mathrm{~s} 2 \mathrm{p}(3 \mathrm{Po}) 4 \mathrm{Po} 5 / 2$ & & 895.4381 & $5.03 \mathrm{E}+02$ & $9.59 \mathrm{E}+07$ & 0.0000 \\
\hline 10 & 3 & 8 & 2 & 1 & 1 & $1 \mathrm{~s}(2 \mathrm{~S}) 2 \mathrm{~s} 2 \mathrm{p}(3 \mathrm{Po}) 2 \mathrm{Po} 1 / 2$ & 908.0482 & 907.7819 & $7.98 \mathrm{E}+12$ & $7.73 \mathrm{E}+12$ & 0.5080 \\
\hline 10 & 3 & 9 & 2 & 1 & 3 & $1 \mathrm{~s}(2 \mathrm{~S}) 2 \mathrm{~s} 2 \mathrm{p}(3 \mathrm{Po}) 2 \mathrm{Po} 3 / 2$ & 908.0482 & 907.8962 & $8.13 \mathrm{E}+12$ & $6.38 \mathrm{E}+12$ & 0.5603 \\
\hline 10 & 3 & 10 & 4 & 1 & 1 & $1 \mathrm{~s}(2 \mathrm{~S}) 2 \mathrm{p} 2(3 \mathrm{P}) 4 \mathrm{P} 1 / 2$ & & 912.3807 & $1.76 \mathrm{E}+09$ & $<5.00 \mathrm{E}+08$ & 1.0000 \\
\hline 10 & 3 & 11 & 4 & 1 & 3 & $1 \mathrm{~s}(2 \mathrm{~S}) 2 \mathrm{p} 2(3 \mathrm{P}) 4 \mathrm{P} 3 / 2$ & & 912.4757 & $1.98 \mathrm{E}+09$ & $6.00 \mathrm{E}+09$ & 0.2481 \\
\hline 10 & 3 & 12 & 4 & 1 & 5 & $1 \mathrm{~s}(2 \mathrm{~S}) 2 \mathrm{p} 2(3 \mathrm{P}) 4 \mathrm{P} 5 / 2$ & & 912.6306 & $2.39 \mathrm{E}+09$ & $3.70 \mathrm{E}+10$ & 0.0607 \\
\hline 10 & 3 & 13 & 2 & 1 & 1 & $1 \mathrm{~s}(2 \mathrm{~S}) 2 \mathrm{~s} 2 \mathrm{p}(1 \mathrm{Po}) 2 \mathrm{Po} 1 / 2$ & & 914.3583 & $9.06 \mathrm{E}+11$ & $7.57 \mathrm{E}+13$ & 0.0118 \\
\hline 10 & 3 & 14 & 2 & 1 & 3 & $1 \mathrm{~s}(2 \mathrm{~S}) 2 \mathrm{~s} 2 \mathrm{p}(1 \mathrm{Po}) 2 \mathrm{Po} 3 / 2$ & & 914.4365 & $7.55 \mathrm{E}+11$ & $7.70 \mathrm{E}+13$ & 0.0097 \\
\hline 10 & 3 & 15 & 2 & 2 & 3 & $1 \mathrm{~s}(2 \mathrm{~S}) 2 \mathrm{p} 2(1 \mathrm{D}) 2 \mathrm{D} 3 / 2$ & 920.4218 & 920.5238 & $4.25 \mathrm{E}+12$ & $1.29 \mathrm{E}+14$ & 0.0319 \\
\hline 10 & 3 & 16 & 2 & 2 & 5 & $1 \mathrm{~s}(2 \mathrm{~S}) 2 \mathrm{p} 2(1 \mathrm{D}) 2 \mathrm{D} 5 / 2$ & 920.4218 & 920.5326 & $4.22 \mathrm{E}+12$ & $1.30 \mathrm{E}+14$ & 0.0314 \\
\hline 10 & 3 & 17 & 2 & 1 & 1 & $1 \mathrm{~s}(2 \mathrm{~S}) 2 \mathrm{p} 2(3 \mathrm{P}) 2 \mathrm{P} 1 / 2$ & & 922.5025 & $1.32 \mathrm{E}+13$ & $2.00 \mathrm{E}+09$ & 0.9998 \\
\hline 10 & 3 & 18 & 2 & 1 & 3 & $1 \mathrm{~s}(2 \mathrm{~S}) 2 \mathrm{p} 2(3 \mathrm{P}) 2 \mathrm{P} 3 / 2$ & & 922.6991 & $1.32 \mathrm{E}+13$ & $4.05 \mathrm{E}+11$ & 0.9702 \\
\hline 10 & 3 & 19 & 2 & 0 & 1 & $1 \mathrm{~s}(2 \mathrm{~S}) 2 \mathrm{p} 2(1 \mathrm{~S}) 2 \mathrm{~S} 1 / 2$ & & 932.4598 & $3.95 \mathrm{E}+12$ & $1.92 \mathrm{E}+13$ & 0.1706 \\
\hline
\end{tabular}

Note.-Table 11 is published in its entirety in the electronic edition of the Astrophysical Journal Supplement. A portion is shown here for guidance regarding its form and content.

TABLE 12

K-Vacancy Transitions in the $\mathrm{Ne}, \mathrm{Mg}, \mathrm{Si}, \mathrm{S}$, Ar, and Ca Isonuclear Sequences

\begin{tabular}{|c|c|c|c|c|c|c|}
\hline$Z$ & $N$ & $k$ & $i$ & $\begin{array}{c}\lambda \\
(0.1 \mathrm{~nm})\end{array}$ & $\begin{array}{c}A(k, i) \\
\left(\mathrm{s}^{-1}\right)\end{array}$ & $g f(i, k)$ \\
\hline 10 & 2 & 4 & 1 & 13.5582 & $3.67 \mathrm{E}+09$ & $3.04 \mathrm{E}-04$ \\
\hline 10 & 2 & 7 & 1 & 13.4470 & $9.87 \mathrm{E}+12$ & $8.04 \mathrm{E}-01$ \\
\hline 10 & 3 & 4 & 2 & 14.1641 & $1.80 \mathrm{E}+11$ & $1.09 \mathrm{E}-02$ \\
\hline 10 & 3 & 4 & 3 & 14.1673 & $3.49 \mathrm{E}+11$ & $2.10 \mathrm{E}-02$ \\
\hline 10 & 3 & 5 & 1 & 13.8471 & $2.69 \mathrm{E}+08$ & $1.54 \mathrm{E}-05$ \\
\hline 10 & 3 & 6 & 1 & 13.8456 & $6.86 \mathrm{E}+08$ & $7.88 \mathrm{E}-05$ \\
\hline 10 & 3 & 8 & 1 & 13.6548 & $7.98 \mathrm{E}+12$ & $4.46 \mathrm{E}-01$ \\
\hline 10 & 3 & 9 & 1 & 13.6531 & $8.13 \mathrm{E}+12$ & $9.09 \mathrm{E}-01$ \\
\hline 10 & 3 & 10 & 2 & 13.8265 & $5.31 \mathrm{E}+08$ & $3.05 \mathrm{E}-05$ \\
\hline 10 & 3 & 10 & 3 & 13.8297 & $2.22 \mathrm{E}+07$ & $1.28 \mathrm{E}-06$ \\
\hline 10 & 3 & 11 & 2 & 13.8251 & $5.52 \mathrm{E}+06$ & $6.34 \mathrm{E}-07$ \\
\hline 10 & 3 & 11 & 3 & 13.8282 & $7.60 \mathrm{E}+08$ & $8.72 \mathrm{E}-05$ \\
\hline 10 & 3 & 12 & 3 & 13.8258 & $1.15 \mathrm{E}+09$ & $1.99 \mathrm{E}-04$ \\
\hline 10 & 3 & 13 & 1 & 13.5566 & $9.00 \mathrm{E}+11$ & $4.96 \mathrm{E}-02$ \\
\hline 10 & 3 & 14 & 1 & 13.5554 & $7.49 \mathrm{E}+11$ & $8.26 \mathrm{E}-02$ \\
\hline 10 & 3 & 15 & 2 & 13.7020 & $3.82 \mathrm{E}+12$ & $4.31 \mathrm{E}-01$ \\
\hline 10 & 3 & 15 & 3 & 13.7051 & $4.25 \mathrm{E}+11$ & $4.79 \mathrm{E}-02$ \\
\hline 10 & 3 & 16 & 3 & 13.7050 & $4.22 \mathrm{E}+12$ & $7.13 \mathrm{E}-01$ \\
\hline 10 & 3 & 17 & 2 & 13.6721 & $8.92 \mathrm{E}+12$ & $5.00 \mathrm{E}-01$ \\
\hline 10 & 3 & 17 & 3 & 13.6752 & $4.32 \mathrm{E}+12$ & $2.42 \mathrm{E}-01$ \\
\hline 10 & 3 & 18 & 2 & 13.6692 & $1.90 \mathrm{E}+12$ & $2.13 \mathrm{E}-01$ \\
\hline 10 & 3 & 18 & 3 & 13.6722 & $1.13 \mathrm{E}+13$ & $1.27 \mathrm{E}+00$ \\
\hline 10 & 3 & 19 & 2 & 13.5236 & $1.21 \mathrm{E}+12$ & $6.67 \mathrm{E}-02$ \\
\hline 10 & 3 & 19 & 3 & 13.5266 & $2.73 E+12$ & $1.50 \mathrm{E}-01$ \\
\hline
\end{tabular}

NotE.-Table 12 is published in its entirety in the electronic edition of the Astrophysical Journal Supplement. A portion is shown here for guidance regarding its form and content. 
MCDF YODA code have given us the opportunity to benchmark the accuracy of the present data sets. This has been useful for sorting out the large discrepancies reported by Palmeri et al. (2003a) for Fe XXI and Palmeri et al. (2006) for S XI with the MCDF data of Chen et al. (1997). Similar discrepancies are found with their data for Ar XIII which, in the light of the good agreement of HFR1 with Biémont et al., are certainly not due to the more formal relativistic representation of the MCDF method. Significant discords of HFR1 with the Auger widths reported by Faenov et al. (1994) for the $\mathrm{Mg}, \mathrm{Si}$, and $\mathrm{S}$ isonuclear sequences and with those by Behar \& Netzer (2002) for levels in the B and $\mathrm{C}$ isoelectronic sequences are difficult to explain. Accuracy ratings of the present $A$-values and Auger widths greater than $10^{13} \mathrm{~s}^{-1}$ are confidently assigned to $15 \%$ and $20 \%$, respectively, except for special cases, e.g., transitions involving K-vacancy levels in the carbon isoelectronic sequence in which strong admixture causes severe departures.

The present radiative and Auger widths will be used in the computations of the $\mathrm{K}$-shell photoionization cross sections of these medium- $Z$ ions which are required in XSTAR for the modeling of interesting spectral features. Future work will involve extension of the present approach to the $\mathrm{Ni}$ isonuclear sequence.

M. A. B. acknowledges partial support from FONACIT, Venezuela, under contract S1-20011000912. This work was funded in part by the NASA Astronomy and Physics Research and Analysis Program. Partial support for C. M. was provided by a travel grant from IVIC and the FNRS of Belgium.
Armour, I. A., Silver, J. D., \& Träbert, E. 1981, J. Phys. B, 14, 3563

Badnell, N. R. 1986, J. Phys. B, 19, 3827 1997, J. Phys. B, 30, 1

Bar-Shalom, A., Klapisch, M., \& Oreg, J. 2001, J. Quant. Spectrosc. Radiat. Transfer, 71, 169

Bautista, M. A., \& Kallman, T. R. 2001, ApJS, 134, 139

Bautista, M. A., Mendoza, C., Kallman, T. R., \& Palmeri, P. 2003, A\&A, 403, 339

. 2004, A\&A, 418, 1171

Behar, E., \& Netzer, H. 2002, ApJ, 570, 165

Beiersdorfer, P., Bitter, M., Hey, D., \& Reed, K. J. 2002, Phys. Rev. A, 66, 032504

Biémont, E., et al. 2000, Phys. Scr., 61, 555

Boroson, B., Vrtilek, S. D., Kallman, T., \& Corcoran, M. 2003, ApJ, 592, 516

Chang, C., \& Cui, W. 2007, ApJ, 663, 1207

Chen, M. H. 1985, Phys. Rev. A, 31, 1449

Chen, M. H., \& Crasemann, B. 1988, At. Data Nucl. Data Tables, 38, 381

Chen, M. H., Reed, K. J., McWilliams, D. M., Guo, D. S., Barlow, L., Lee, M., \& Walker, V. 1997, At. Data Nucl. Data Tables, 65, 289

Cocke, C. L., Curnutte, B., \& Randall, R. 1974, Phys. Rev. A, 9, 1823

Cowan, R. D. 1981, The Theory of Atomic Structure and Spectra (Berkeley: Univ. California Press)

Crespo López-Urrutia, J. R., Beiersdorfer, P., \& Widmann, K. 2006, Phys. Rev. A, 74, 012507

Davis, W. A., \& Marrus, R. 1977, Phys. Rev. A, 15, 1963

Deslattes, R. D., Kessler, E. G., Indelicato, P., de Billy, L., Lindroth, E., \& Anton, J. 2003, Rev. Mod. Phys., 75, 35

Dohmann, H. D., \& Mann, R. 1979, Z. Phys. A, 291, 15

Drake, G. W. F. 1979, Phys. Rev. A, 19, 1387

Eissner, W., Jones, M., \& Nussbaumer, H. 1974, Comput. Phys. Commun., 8, 270

Faenov, A. Ya., Pikuz, S. A., \& Shlyaptseva, A. S. 1994, Phys. Scr., 49, 41

García, J., Mendoza, C., Bautista, M. A., Gorczyca, T. W., Kallman, T. R., \& Palmeri, P. 2005, ApJS, 158, 68

Gorczyca, T. W., Kodituwakku, C. N., Korista, K. T., Zatsarinny, O., Badnell, N. R., Behar, E., Chen, M. H., \& Savin, D. W. 2003, ApJ, 592, 636

Groeneveld, K. O., Mann, R., Nolte, G., Schumann, S., \& Spohr, R. 1975, Phys. Lett. A, 54, 335

\section{REFERENCES}

Hagelstein, P. L., \& Jung, R. K. 1987, At. Data Nucl. Data Tables, 37, 121 Haselton, H. H., Thoe, R. S., Mowat, J. R., Griffin, P. M., Pegg, D. J., \& Sellin, I. A. 1975, Phys. Rev. A, 11, 468

Hellmann, H., \& Träbert, E. 1985, Nucl. Instr. Meth. Phys. Res. B, 9, 611

Holczer, T., Behar, E., \& Kaspi, S. 2007, ApJ, 663, 799

Hubricht, G., \& Träbert, E. 1987, Z. Phys. D, 7, 243

Kaastra, J. S., \& Mewe, R. 1993, A\&AS, 97, 443

Kallman, T. R., Palmeri, P., Bautista, M. A., Mendoza, C., \& Krolik, J. H. 2004, ApJS, 155, 675

Kaspi, S., et al. 2002, ApJ, 574, 643

Knystautas, E. J., \& Druetta, M. 1985, Phys. Rev. A, 31, 2279

Kramida, A. E., \& Buchet-Poulizac, M.-C. 2006, Eur. Phys. J. D, 39, 173

Lee, J. C., et al. 2001, ApJ, 554, L13

Mendoza, C., Kallman, T. R., Bautista, M. A., \& Palmeri, P. 2004, A\&A, 414, 377

Palmeri, P., Mendoza, C., Bautista, M. A., García, J., \& Kallman, T. R. 2006, Radiant Phys. Chem., 75, 1465

Palmeri, P., Mendoza, C., Kallman, T. R., \& Bautista, M. A. 2002, ApJ, 577, L119

. 2003a, A\&A, 403, 1175

Palmeri, P., Mendoza, C., Kallman, T. R., Bautista, M. A., \& Meléndez, M. 2003b, A\&A, 410, 359

Ralchenko, Yu., et al. 2007, NIST Atomic Spectra Database, version 3.1.2 (Gaithersburg: NIST), http://physics.nist.gov/asd3

Safronova, U. I., \& Shlyaptseva, A. S. 1999, Phys. Scr., 60, 36

Stefanelli, G. S., Beiersdorfer, P., Decaux, V., \& Widmann, K. 1995, Phys. Rev. A, 52, 3651

Träbert, E., Beiersdorfer, P., Brown, G. V., Smith, A. J., Utter, S. B., Gu, M. F., \& Savin, D. W. 1999, Phys. Rev. A, 60, 2034

Vainshtein, L. A., \& Safronova, U. I. 1978, At. Data Nucl. Data Tables, 21, 49 1980, At. Data Nucl. Data Tables, 25, 311

Varghese, S. L., Cocke, C. L., \& Curnutte, B. 1976a, Phys. Rev. A, 14, 1729 Varghese, S. L., Cocke, C. L., Curnutte, B., \& Seaman, G. 1976b, J. Phys. B, 9 , L387

Watanabe, S., et al. 2006, ApJ, 651, 421 